
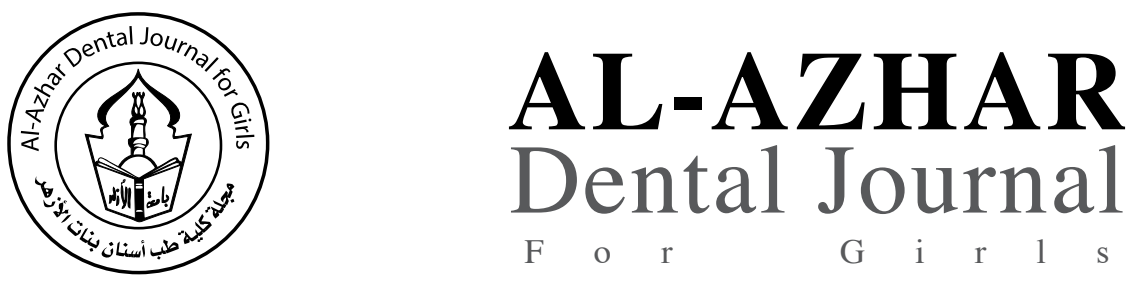

The Official Publication of The Faculty of Dental Medicine For Girls, Al-Azhar University Cairo, Egypt.

ADJ-for Grils, Vol. 3, No. 2, April (2016) — PP. 111:118

\title{
Influence of Mixing Methods, Special Trays and Storage Time on the Accuracy of two Elastomeric Impressions
}

\author{
Noha O. El Tawiel ${ }^{(1)}$, Mohamed M. El-Hossary ${ }^{(2)}$ and Eman A. Essam ${ }^{(3)}$
}

Codex : 04/1604

dentaljournal.forgirls@yahoo.com

\begin{abstract}
This in-vitro study investigated the dimensional accuracy and stability of polyether and polyvinylsiloxane impression materials. The study included the effect of mixing methods where conventional mix versus auto-mixing technique, the effect of special tray on the accuracy of impression and pouring the impressions at different times of storage (1hour, 48 hours and 7 days). Impressions were made for a master model cast of mandibular arch with two prepared abutments; right second premolar and right second molar with a shoulder finish line made as to give a definite margin that was easily defined where there was an occlusal shoulder and gingival shoulder used for occlusogingival (O-G) measurements. There was an occlusal holes made on occlusal surface of both teeth to be used as a reference points for inter-abutment distance record of the prepared abutments. All dimensions were measured on casts retrieved from impressions made on master model. The impressions were then poured with extra-hard stone into stone casts, according to times under investigations. The results revealed that the polyether elastomeric impression material presented a higher degree of accuracy than vinylpolysiloxane. The automixing technique showed better results than conventional mixing, while the light- cured special tray presented slightly better dimensions. Also, the one- hour pouring time presented the best dimensions, while two- days and sevendays pouring times had almost the same dimensions.
\end{abstract}

\section{INTRODUCTION}

Impression making is an important step in the complex process of fabricating a well- fitting indirect prosthetic restoration. Accuracy of the impression material in terms of both dimensional accuracy and details reproduction is an essential pre-requisite for successful impression.
Elastomeric impression materials,

Special trays,

Dimensional change.

1. B.D.S Tanta University, 2005, Dentist in Al Zahraa Hospital.

2. Professor of Crowns and Bridges, Faculty of Dental Medicine "Boys" Cairo,Al-Azhar University.

3. Associate Professor, Crowns and Bridges Department, Faculty of Dental Medicine, Al-Azhar University, Girls’ Branch. 
When hand-mixing impression material is difficult to blend and incorporate the catalyst with the base, it is virtually impossible to achieve a homogenous voidfree mix. Several manufacturers offer impression materials in pre-packed cartridges to which a disposable mixing tip is attached, then inserted in a caulking gunlike device. The base and the catalyst are extruded into the mixing tip, where they progress to the end of the tube .The homogeneously incorporated material can be directly placed on the prepared teeth and the impression tray. This system has the advantage of eliminating the variable of hand mixing on pads and produces fewer voids in the impression. However, the heavy body polyethers are not available in this system because they are difficult to extrude from the cartridge when this system is used .Therefore the mixing of these high viscosity materials has been simplified with the electric mechanical mixing system. This was proven to produce fewer voids in the impression material and saves time and effort ${ }^{(2,3)}$.

The dimensional stability and the accuracy of elastomeric impression materials are maximized when a custom tray is used. Dies obtained by using a custom tray are more accurate than those obtained by using stock trays. Light curing resin can be used for construction of an intraoral custom tray ${ }^{(4)}$.

The rate of shrinkage of elastomeric impressions is not uniform during the twenty-four hours after removal from the mouth. In general, about half the shrinkage is observed during the first hours after removal. Although in air the elastomeric impression materials are much more stable than hydrocolloid products ${ }^{(5)}$. Hence, the purpose of this study was to evaluate the effect of mixing methods (whether conventional or auto-mixing), special tray material and storage time (1 hour, 48 hours, 7 days), on the accuracy of working casts produced from polyvinylsiloxane and polyether elastomeric impressions. The null hypothesis was that there would be no significant differences between the two tested materials ${ }^{(6,7)}$.

\section{MATERIALS AND METHODS}

To conduct this study, a master model (El Banna Dental Product INC., Alex- Egypt) of mandibular arch with missing lower right first molar and two abutments; right second premolar and right second molar was used. Both abutments received full-veneered preparations with cervical shoulder finish lines of thickness $1.5 \mathrm{~mm}$, Fig (1).

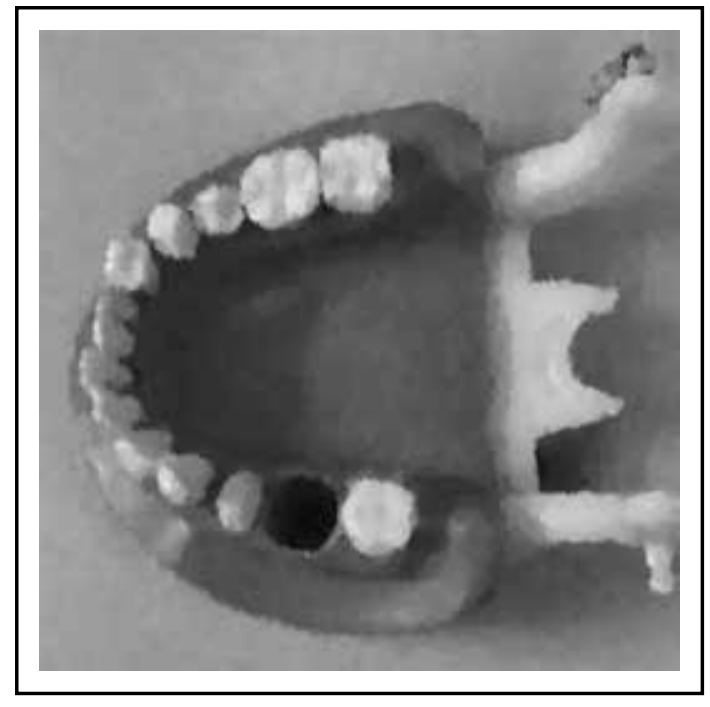

Fig. (1) Abutment preparations

The inter-abutment distance (I-A) between the second premolar and second molar was measured between occlusal holes made on both teeth as reference points, these occlusal holes were made using tapered stone with flat end and the holes were made at the central fossae on the occlusal surface. An additional occlusal shoulder was prepared on the buccal surface of the premolar abutment $5.5 \mathrm{~mm}$ from the cervical shoulder and this was set to be the occluso-gingival measurement $(\mathrm{O}-\mathrm{G})$ on the master model. These two dimensions I-A and $\mathrm{O}-\mathrm{G}$ were to be the parameters of accuracy measurements later on in the study.

\section{Sample grouping}

\section{Samples were divided into two main groups according to impression type:}

Group A: Vinylpolysiloxane impression (3M ESPE, Germany), (number of samples=60). 
Group B: Polyether impression (Impregum, 3M ESPE,Germany), (number of samples=60). Then, each group was divided into 2 sub-groups according to mixing method.

Subgroup I: Auto- mixing (number of samples=30) \& Sub-group II: Conventional mixing (number of samples=30). These subgroups were further divided into two divisions according to type of special tray; Division 1: light cure special tray(Ivoclar vivadent) (number of samples=15).

Division2: auto- cure special tray (Pekatray, Bayer dental,Leverkusen ) (number of samples=15).

Then, these divisions were divided to three subdivisions according to the storage timing; Subdivision a: 1 hour storage (number of samples=5). Sub-division b: 48hours storage (number of samples=5). Sub-division c: 7 days storage (number of samples $=5$ ).

Auto-cured and light cured special trays were made to be uniformly with $2 \mathrm{~mm}$ clearance between the tray and master model. Then an alginate impression was made for the master model with the spacer. The impression was poured with improved stone into a stone cast. The stone cast served in producing the custom trays each time with the same clearance space.

\section{Auto- cured special trays construction}

The autopolymerizing acrylic resin was proportioned and mixed according to the manufacturer's directions. When the dough stage was reached, it was adapted and shaped over the stone cast. In this way a constant and uniform interface thickness between the master model and the acrylic tray was obtained. Festoons were uniformly cut at the border of the tray using a carbide bur. Festoons permitted the escape of excess material, allowing complete seating of the tray, they also acted as a secondary aid for retention of the impression material inside the tray other than the tray adhesive. The trays were not used for at least two hours after construction to minimize any effects that could result from shrinkage of the tray itself. Adhesive provided by the respective manufacturer was thinly applied with a brush on the trays and allowed to dry for at least 30 minutes before the impressions were made to allow the volatile ingredients of the adhesive to evaporate.

\section{Light- cure special trays construction}

Light-cure special tray consists of acrylated oligomers, glass fillers, dimethacrylate, silicondioxide, catalyst and pigments. After blocking out undercut areas, the model was soaked in and isolated with ivoclar separting fluid. The light tray was moulded onto the model. Adhesion of material to fingers was prevented with a little water. The functional edge was cut to length with a sharp instrument and excess was removed. The surplus material was used to build the tray grip. The completed tray was polymerized in polymerization unit(Bre.Lux power unit). The material was cured from both sides without vacuum for 3-5 minutes. Finishing was done immediately after curing. Optimum physical properties were achieved after a polymarization time of $5 \mathrm{~min}$ utes. Festoons were made as previously described.

Impression Making: All materials were mixed and used within the working time recommended by the manfactures .The setting time of each impression was constant for a given technique and material.

\section{Impression making for polyether (Impregum) group:}

It's a medium- viscosity; (monophase technique):

a) Conventional mixing: (Hand mixing): two equal lines of impression paste base and catalyst were spread on glass slap and mixing was done by metal spatula in circular strokes for producing homogenous, void-free mixed impression. Impression material was then loaded into the tray which was then centered over the model and seated until full seating, with the escape of excess material till the created stoppers that were done to ensure uniform seating. Then, trays were steadily held with no applied pressure till polymerization and setting were completed. 
b) Automatic mixing: Using the pentamix automatic mixing unit (3M ESPE, Germany). It was used with both types of impression materials, Impregum and Express XT penta Putty. Impregum penta is a medium-bodied consistency polyether impression material for the penta mix machine. The mixing ratio is 5 volumes base paste: 1 volume catalyst. It was supplied as polybags that were sealed with a pentamatic sealing cap. This cap automatically opens the poly bags once sufficient pressure is established by the plunger of the penta mix. Impregum penta was placed in the designated cartridge. The pentamatic sealing cap must be used with penta mixing red tips. The first $3 \mathrm{~cm}$ of paste was discarded from newly filled cartridge prior to the first use for impression taking. The color of the dispensed paste must be uniform. Dosing and mixing were performed automatically in the penta mix. Setting time from the start of mixing (entry of paste into the mixing tip) was 6 minutes.

\section{II- Impression making for vinylpolysiloxane (Express) group:}

a) Automatic mixing: using the penta mix automatic mixing unit as previously described The Express XT penta putty material was used with Express XT regular body to produce a simultaneous putty wash (1-step technique) impression. The garant dispenser is used with all express wash materials. The base and the catalyst are extrused into the garant disposable mixing tip, where mixing occurs as they progress to the end of the tube.

b) Conventional (hand) mixing: Express STD putty impression material was used. It is designed to be combined with Express wash materials. It has 1- minute of room temperature working time. The mixing ratio is 1 scoop of base paste: 1 scoop of catalyst paste. The base and catalyst were mixed with fingertips until a homogeneous color is achieved (30 seconds). The mixed putty is then placed into the adhesive- coated tray. The Express XT regular body was also prepared to produce a simultaneous putty- wash (1- step technique) impression as previously described.
Storage of impressions: There were three storage timings in this study: after one hour, forty eight hours, and seven days. All impressions were kept in labeled plastic boxes according to their groups and sub-groups as were mentioned in the sampling; in dry environmental conditions and room temperature till they were poured at their specific timings.

Pouring the impressions: Type IV extra hard stone(GC FuJirocic EP type IV dental stone) was mixed and poured in the impression using a vibrator to decrease probability for air bubbles.The produced casts were left to set for the time instructed by the manufacturer (20 minutes). Then the casts were removed from the impressions, Fig(2).

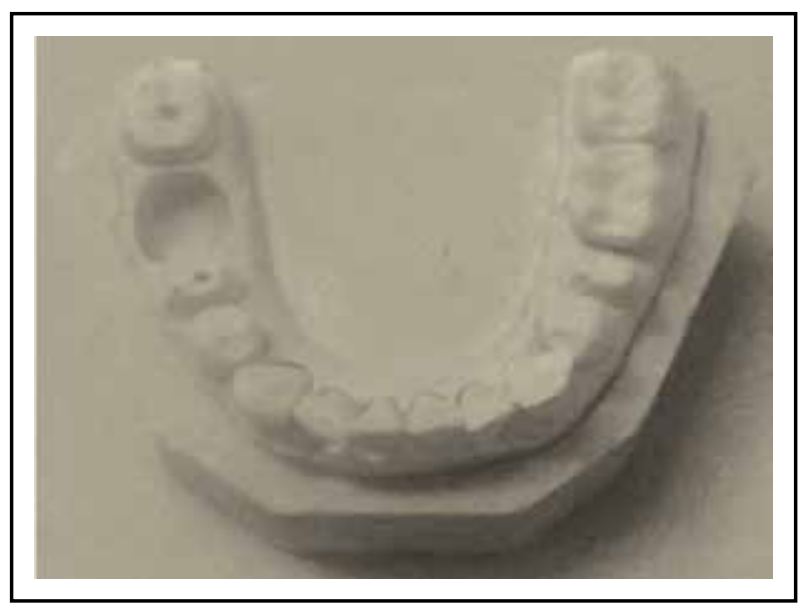

Fig. (2) AStone Cast

\section{Testing:}

The accuracy of all specimens through the I-A and $\mathrm{O}-\mathrm{G}$ were determined by their deviation from the master model measures; by measuring it via stereomicroscope measures. For each cast, three photomicrographs were undertaken by CCD digital camera (DP10, Olympus- Japan) attached to zoom stereo microscope (Olympus SZ- PT- Japan). The magnification used was x6.7. The I-A was linearly measured by splitting this distance into two halves, one between the molar and a drawn line and the other between the premolar and the line. Then the two measures were added. O-G linear measurement 
was measured vertically between the 2 finishing lines. These linear measurements were automatically calculated using the image analysis software (Image ware, Image J1.37v, USA). Measurements were recorded and tabulated for statistical analysis and compared with the original dimensions on master model, Fig(3).

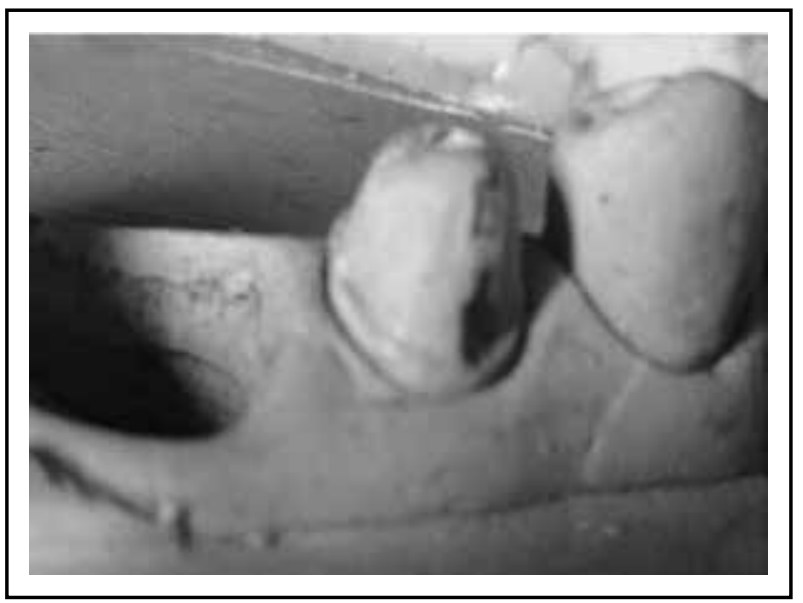

Fig. (3) O-G measurement

\section{RESULTS}

Data were presented as mean and standard deviation (SD) values. Repeated measures ANOVA followed by Newman keuls post hoc test was used for pair-wise comparisons to compare between main measurements of different techniques and the master model. Differences between master model measurements (intrabutment distance, height) and stone model measurements represent the dimensional changes.

Statistical analysis was performed using Aasistat 7.6 statistics software for Windows (Campina Grande, Paraiba state, Brazil). P values $\leq 0.05$ are considered to be statistically significant in all tests.

\section{Effect of mixing technique}

Total percentage change in stone models obtained from automix was $(6.01433 \%)$, which was lower than the value from traditional mix $(8.634648 \%)$.
There was statistically significant $(\mathrm{p}<0.05)$ difference between auto and traditional mix as indicated by t-test. Table(1) \&Fig (4).

Table (1) Comparison between dimensional change $\%$ of both mixing techniques

\begin{tabular}{|c|c|c|c|c|}
\hline Variale & Group & Mean & \pm SD & $\begin{array}{c}\text { t-test } \\
(\text { P value })\end{array}$ \\
\hline \multirow{2}{*}{ Mixing } & Automix & 6.01433 & 3.036847 & \multirow{2}{*}{$0.0242 *$} \\
\cline { 2 - 5 } & Traditional mix & 8.634648 & 3.345088 & \\
\hline
\end{tabular}

$n s$; non-significant $(p>0.05)$

*: Significant at $P \leq 0.05$,

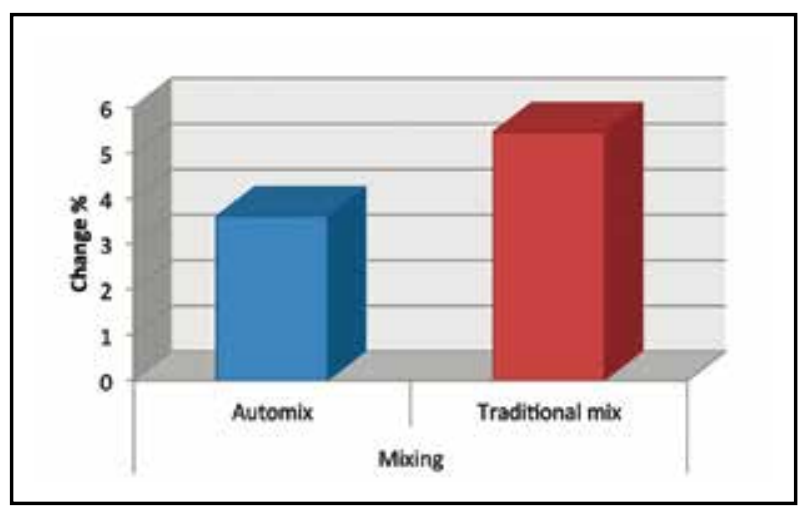

Fig. (4) Chart comparing between dimensional change $\%$ mean values for both mixing techniques

\section{Effect of tray type:}

Total percentage change in stone models obtained from LC tray was (7.946875\%), which was lower than the value from AC tray (8.919722\%). There was statistically non-significant $(\mathrm{p}>0.05)$ difference between LC and AC trays as indicated by t-test. Table(2) \&Fig(5).

Table (2) Comparison between dimensional change $\%$ of both tray types

\begin{tabular}{|c|c|c|c|c|}
\hline Variale & Group & Mean & $\pm \mathrm{SD}$ & $\begin{array}{c}\text { t-test } \\
(\mathrm{P} \text { value })\end{array}$ \\
\hline \multirow{2}{*}{ Tray type } & LC tray & 7.946875 & 0.66783 & \multirow{2}{*}{$0.0632 \mathrm{~ns}$} \\
\cline { 2 - 4 } & AC tray & 8.919722 & 0.732464 & \\
\hline
\end{tabular}

$n s$; non-significant $(p>0.05)$

*: Significant at $P \leq 0.05$, 


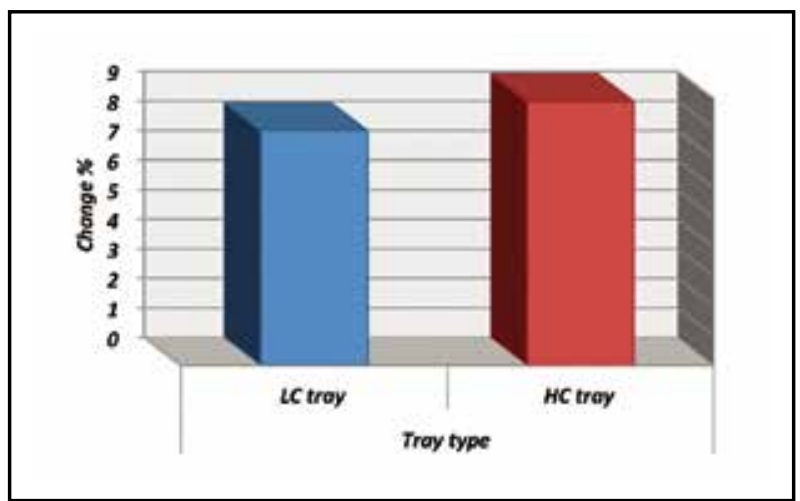

Fig. (5) Chart comparing between dimensional change $\%$ mean values for tray types

\section{Effect of time:}

The highest total percentage change in stone models obtained after 7 days $(9.979212 \%)$ followed by $48 \mathrm{hrs}(9.304308 \%)$ while the lowest total percentage change in stone models was obtained after $1 \mathrm{hr}(6.62236 \%)$. There was statistically non-significant ( $p>0.05$ ) difference between different pouring times as indicated by ANOVA-test. Table(3), Fig(6).

Tab. (3) Comparison between dimensional change $\%$ of different pouring times

\begin{tabular}{|c|c|c|c|c|}
\hline Variale & Group & Mean & $\pm \mathrm{SD}$ & $\begin{array}{c}\text { ANOVA-test } \\
\text { (P value) }\end{array}$ \\
\hline \multirow{3}{*}{$\begin{array}{c}\text { Pouring } \\
\text { time }\end{array}$} & $1 \mathrm{hr}$ & 6.62236 & 3.035905 & \multirow{3}{*}{$0.1213 \mathrm{~ns}$} \\
\hline & $48 \mathrm{hr}$ & 9.304308 & 2.421376 & \\
\hline & $7 \mathrm{~d}$ & 9.979212 & 2.116319 & \\
\hline
\end{tabular}

$n s ;$ non-significant $(p>0.05)$

*: Significant at $P \leq 0.05$,

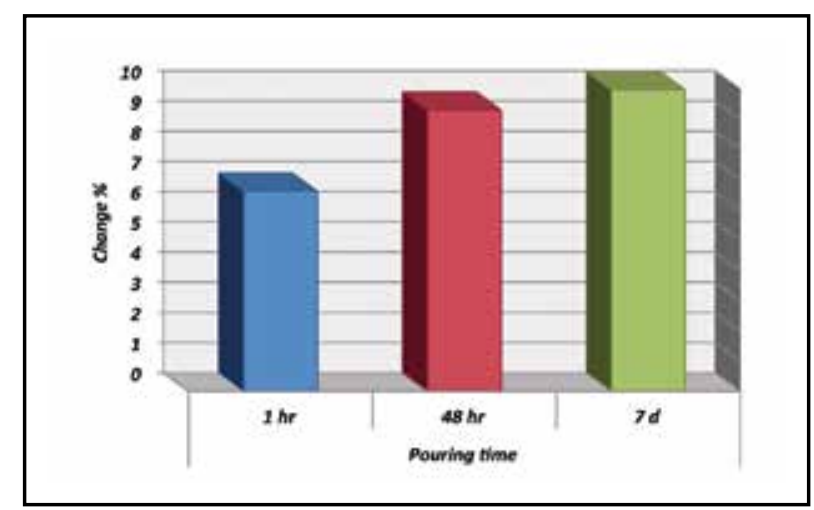

Fig. (6) Chart comparing between dimensional change $\%$ mean values for different pouring times
Total percentage change in stone models obtained from PE material was (10.7934\%), which was lower than the value from VPS material $(11.14278 \%)$. There was statistically non-significant ( $\mathrm{p}>0.05$ ) difference between PE and VPS materials as indicated by t-test. Table (4), Fig(7).

Tab. (4) Comparison between dimensional change $\%$ of both materials

\begin{tabular}{|c|c|c|c|c|}
\hline Variale & Group & Mean & \pm SD & $\begin{array}{c}\text { t-test } \\
(\mathrm{P} \text { value })\end{array}$ \\
\hline \multirow{2}{*}{ Material } & PE & 10.7934 & 1.424638 & \multirow{2}{*}{$0.7961 \mathrm{~ns}$} \\
\cline { 2 - 4 } & VPS & 11.14278 & 1.904549 & \\
\hline
\end{tabular}

$n s$; non-significant $(p>0.05)$

*: Significant at $P \leq 0.05$,

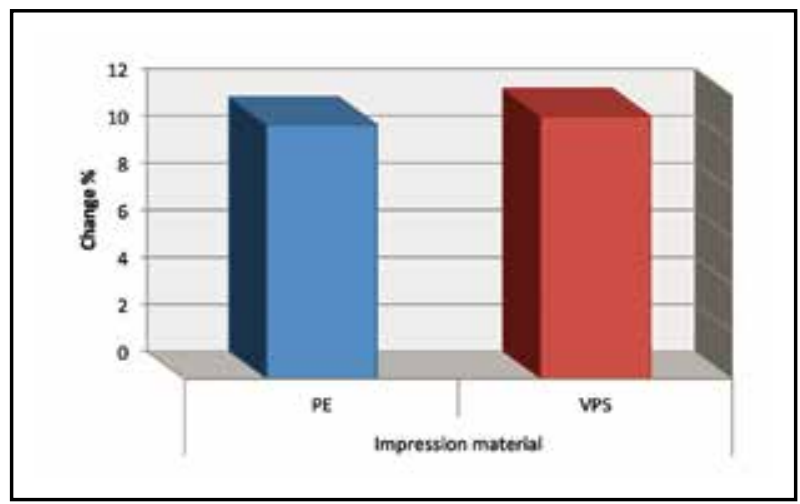

Fig. (7) Chart comparing between dimensional change $\%$ mean values for both materials

\section{DISCUSSION}

In the present investigation, the impression materials selected were the most accurate brands of elastomeric impression materials, polyether and polyvinylsiloxane. Single mix impression technique was reported as it produces the most accurate impression as evidenced by ciesco et $\mathrm{l}^{(1)}$ and lacy et al. ${ }^{(2)}$

Custom trays are more intimately adapted to the master model. Requiring less impression material when compared to stock impression trays, ${ }^{(3)}$ this was reported to improve the accuracy of the impression. 
Also, the use of custom trays decreased the bulk of the material used and its uniformty around the abutment that was reported to increase the accuracy of the impression ${ }^{(4-6)}$ through minimizing the dimensional changes caused by the polymerization shrinkage. Rigid custom impression trays were used as to minimize the deformation of impression materials and standardize the forces applied while removing it from the cast.

The effect of the time of pour on the dimensional stability of polyether and poly vinylsiloxane was investigated. Results indicated that the immediate pour of impression into stone resulted in the most dimensionaly stable results, with a highly significant value than other pouring times (48hrs and the 7 days pour).

It was noted that the immediate pouring of polyvinylsiloxanes resulted in porous surface of stone casts, which explained by Craig, R.G. ${ }^{(7)}$ and Dhuru V.B. ${ }^{(8)}$ is due to hydrogen release in the first hour pouring .

Newan and szojka, ${ }^{(9)}$ in their study on the dynamics of gas production in poly-vinylsiloxane and its effect on the surface quality of the cast made, stated that gas production was related to higher viscosity polyvinylsiloxane.

Ciesco et al, ${ }^{(10)}$ agreed with the results in this study concerning polyether. However they indicated that polyvinylsiloxane is more dimensionally stable when it was poured immediately, however the problem of hydrogen release was not mentioned in the study.

Also Williams PT, ${ }^{(1)}$ confirm with results in this study concerning polyether while they disagreed with results regarding poly vinylsiloxanes, as they stated that immediate pouring had superior results.

Meanwhile, results in this study agree with that of Zaki A, ${ }^{(12)}$ she stated that pouring times recommended by the manfacturer proved to be the most convenient with materials used, this means that immediate pouring for polyethers and the one hourpour for polyvinylsiloxanes were recommended.
Ruceda et al, ${ }^{(13)}$ stated that there were no significant dimensional difference in cast produced from different pouring times, which was the same results concluded in Andree P et al study. ${ }^{(14)}$

Nonetheless, with regards to the effect of pouring sequence in this study on the dimensional stability of polyethers and polyvinylsiloxane, highly signficant statisitical results favoured the one- hour pour into stone rather than delayed pouring.

The polyvinylsiloxane and polyether are the most accurate impression materials used in fixed prosthodontics work and that's may be due to the less polymerization shrinkage of the addition setting reaction and to the absence of reaction by -product which when evaporate increases the shrinkage. Moreover, the use of a tray adhesive directs the polymerization shrinkage toward the tray walls thus decreasing the shrinkage effect.

The effect of using mechanical- mixing systems versus hand mixing was studied by Xavier lepe, ${ }^{(15)}$ he stated that mechanically mixed impression materials were more compact with less voids.

Dimensional accuracy of the materials is considered to depend on the bulk of the elastic material, that is the distance from the inner surface of the trays to the surface of the impression. Creating occlusal stops facilitated a standard points at which the impression seating was standardized and even thickness or bulk of impression was ensured, as accuracy is dependent on material volume used. ${ }^{(16)}$

However, the null hypothesis in the present investigation was rejected, as polyethyer impression material demonstrated better dimensional accuracy records than vinylpoylsiloxane

\section{CONCLUSIONS}

From the results of the present investigation, the following can be concluded:

1. The polyether elastomeric impression material presented a higher degree of accuracy than vinylpolysiloxane. 
2. The automixing technique showed better results than conventional mixing.

3. The light- cured special tray presented slightly better dimensions than auto- cured.

4. The one- hour pouring time presented the best dimensions, while two- days and seven-days pouring times had almost the same dimensions.

\section{CLINICAL RECOMMENDATION}

- Polyether impression material with automixing technique using light- cure special trays and one- hour pouring time would produce satisfactory impression.

- Nowadays, however, there are too many newly introduced commercial products with improved physical and chemical properties. The clinician has to choose the best through his scientific knowledge and clinical experience.

\section{REFERENCES}

1. Ciesco J. N. , Malone W.F.P. , Sandrik J.L. , and Manzur B Comparsion of Elastomeric impression materials used in fixed prosthodontics .J prosthdent 45: 89-94,1981.

2. Lacy AM, Fukui H, Bellman T.,and Jendresen MD :Time dependant accuracy of elastomer impression materials ,part II polyether ,polysulfide and polyvinylsiloxane $\mathbf{J}$ prosth Dent 45:329.

3. Comparsion of Elastomeric impression materials used in fixed prosthodontics .J prosthdent 45: 89-94,1981:.

4. Sudukh Th , B.Keith M., M. Thomas B ., David T. and Carl J.Andres ; Andress : Dimensional Accuracy of dental casts influence of tray material, Impression material and time $\mathbf{J}$ prosthodont 2002, 11: 98-102.

5. Reisbick MH and Matyas J. The accuracy of highly filled elastomeric impression materials. J Prosthet Dent 1975; 33:67-72.
6. Sherfudhin $\mathrm{H}$ and Abdulla MA. Preparation of void-free casts from vinyl polysiloxane impressions. J Dent. 1996 Jan-Mar;24(1-2):95-8.

7. Craig RG. Restorative Dental Materials, ed 8. St Louis, CV Mosby Co, 2006, p 330.

8. Dhuru VB ,Asgharia MK, Mager JC , Hassan K, Surface porosity of stone cast made from polyvinylsiloxane impression material . Oper Dent 1986;11: 3-7.

9. Newan S.M. and Szojka F.: Gas production by vinylpolysiloxane. (IADR Abstracts 816, 1986).

10. Ciesco J. N. , Malone W.F.P. , Sandrik J.L. , and Manzur B: Comparsion of Elastomeric impression materials used in fixed prosthodontics .J prosthdent, 1981; 45: 89-94.

11. Williams PT, Jackson DG, Bergman W. An evaluation of the time- dependent dimensional stability of eleven elastomeric impression materials J Prosthet Dent. 1984 Jul; 52(1):120-5.

12. Zaki A, Purk JH ,Tira DE ,Eick JP :The effects of different storage conditions on polyether and polyvinylsiloxane impression J Am Dent Associ, 1998;129: 1014-21.

13. Rueda L , Sy Munoz J ,Naylor W, Goodacre C and Swartz M.The effects of using custom or stock trays on the accuracy of gypsum casts . Int J prosthodent 1996; 9:367373.

14. Andree Piwowarczyk, Peter OHH, Alfred Buchler, Hans- Christoph Lauer, Andrea Hoffmann: In vitro study on the dimensional accuracy of selected materials for monphasicelastic impression making". Int J Prosthodont International Journal prosthodont 2002; 15: 168-174.

15. Xavier Lepe, Glen H. Johnson, John C. Berg, and Tarc. Aw: Effect of mixing technique on surface characteristics of impression materials. Jprosthet Dent 1998; 79: 495-502.

16. Andree Piwowarczyk, Peter $\mathrm{OHH}$, Alfred Buchler, Hans- Christoph Lauer, Andrea Hoffmann: In vitro study on the dimensional accuracy of selected materials for monphasicelastic impression making". Int J Prosthodont International Journal prosthodont 2002; 15: 168-174. 